\title{
Acute Anaemia Induced by Ancylostomosis in a Puppy and its Successful Therapeutic Management - A Case Report
}

\author{
R. Jasmin ${ }^{1}$, M, Balagangatharathilagar* ${ }^{* 1}$, R. Edith ${ }^{2}$ and S. Prathaban ${ }^{1}$ \\ ${ }^{1}$ Department of Veterinary Clinical Medicine, ${ }^{2}$ Department of Veterinary Parasitology, \\ Madras Veterinary College, Chennai- 600007 \\ Tamil Nadu Veterinary and Animal Sciences University \\ *Corresponding author
}

\section{A B S T R A C T}

K e y w o r d s
Ancylostomosis,
Anaemia, Blood
transfusion,
Anthelminthic.

Keywords

Ancylostomosis transfusion, Anthelminthic.

Article Info

10 April 2020

10 May 2020

\begin{abstract}
A 6 month old Labrador pup was presented to the Critical Care Unit of Madras Veterinary College Teaching Hospital with the history of anorexia, vomiting and diarrhea for two days. Clinical examination revealed poor hair coat, blanched mucous membranes and hemorrhagic diarrhoea. Hematological and serum biochemical parameters showed severe anaemia, leukocytosis and hypoproteinemia. Faecal floatation examination revealed eggs of Ancylostoma caninum. The pup was stabilized with whole blood transfusion, fluids, antibiotics, amino acid injections, hematinics and treated with anthelminthic medications. The animal responded well to the treatment.
\end{abstract}

\section{Introduction}

Ancylostomosis is a hook worm disease caused by Ancylostoma caninum in dogs which is characterized by anorexia, anaemia, weakness, rough hair coat and haemorrhagic enteritis. The Ancylostoma spp. are voracious blood sucking hook worms and also characterized by 3 pairs of ventral teeth and a pair of dorsal teeth in the head end causing bleeding ulcers at the site of bite and also the due to the anticoagulant action of the buccal secretions of the worm. Adult worms lay eggs in small intestine which are excreted through the faeces. The eggs of Ancylostoma spp are oval in shape with thin shelled and segmented yolk. In a suitable environment, eggs then hatch into $\mathrm{L}_{1}$ larvae and undergo two moults into $\mathrm{L}_{2}$ and $\mathrm{L}_{3}$ (Bowman et al., 2010). Infective stage larvae $\left(\mathrm{L}_{3}\right)$ ingested by the 
dogs moults into $\mathrm{L}_{4}$ and mature to adult in the small intestine of dogs. The main routes of transmission are ingestion of infective stage, skin penetration, prenatal and trans-mammary transmission. Yavuzer et al., (2010) diagnosed a case of hookworm-related cutaneous larva migrans in woman and treated her successfully with albendazole indicates the zoonotic potential of the Ancylostoma spp. This paper reports a case of acute anaemia induced by ancylostomosis in a pup and its therapeutic management.

\section{Case history and observations}

A six month old intact male Labrador Retriever weighing $15 \mathrm{~kg}$ was brought to the Critical Care Unit of the Madras Veterinary College Teaching Hospital with the history of anorexia, vomiting and haemorrhagic enteritis for two days. The pup was vaccinated and had never been dewormed earlier.

Clinical examination revealed poor hair coat, blanched mucous membranes (Fig.1), palpable lymph nodes, tachypnea, tachycardia and hemorrhagic enteritis (Fig.2). Hematological findings included anemia (Hemoglobin of $2.8 \mathrm{~g} / \mathrm{dL}$, packed cell volume of $4.2 \%$ and total erythrocyte count of 1.04 million cells per cu.mm), leukocytosis (WBC Count 27,600 per cu.mm) and neutrophilia.

All Serum biochemical parameters were within normal reference ranges except hypoproteinemia $(4.8 \quad \mathrm{~g} / \mathrm{dL}) \quad$ and hypoalbuminemia (1.7 g/dL). Parvo viral enteritis was ruled out through polymerase chain reaction (PCR) assay of the faecal sample.

Ancylostomosis was confirmed by the presence of typical Ancylostoma spp. eggs in the faecal sample of the pup through standard faecal examination technique. (Fig 3).

\section{Results and Discussion}

Treatment approach in this case involved anthelmintic therapy and supportive care. The dog was treated with broad spectrum anthelmintic tablet containing pyrantel, fenbendazole and praziquantel. Broad spectrum antibiotic therapy was given with combination of amoxicillin and potassium clavulanate at $12.5 \mathrm{mg}$ per $\mathrm{kg}$ BID and metronidazole at $10 \mathrm{mg}$ per $\mathrm{kg}$ BID for 5 days. Supportive care included were fluid therapy, hematinics, amino acid supplements and blood transfusion. Around $350 \mathrm{ml}$ of whole fresh blood was transfused after cross matching to relieve severe anaemia (Fig 4). The pup responded well to the treatment.

Ancylostomosis is a very common helminthiasis in dogs under 1 year of age but sometime causes severe fatal anemia in puppies. Adult A. caninum live in the small intestine where they attach themselves with its buccal capsule containing three pairs of ventral teeth and one pair of dorsal teeth. They feed on the dog's blood, secrete anticoagulents and ingest blood from multiple bite sites. A. caninum are voracious blood sucker and consumes in the range of 0.01 to $0.09 \mathrm{ml}$ of blood per worm per day (Jubb et al., 2016).

The predominant anticoagulant serine protease inhibitor isolated from the adult $A$. caninum hookworms which are responsible for the pathogenesis of hookworm associated blood loss and anemia (Cappello et al., 1995). Don et al. (2004) identified a pore-forming hemolysin from the hookworm, A. caninum. Barakat et al. (2012) recorded the in vivo endoscopic imaging of ancylostomosisinduced gastrointestinal bleeding which showed varying small intestinal injuries resulting in luminal blood pools. 


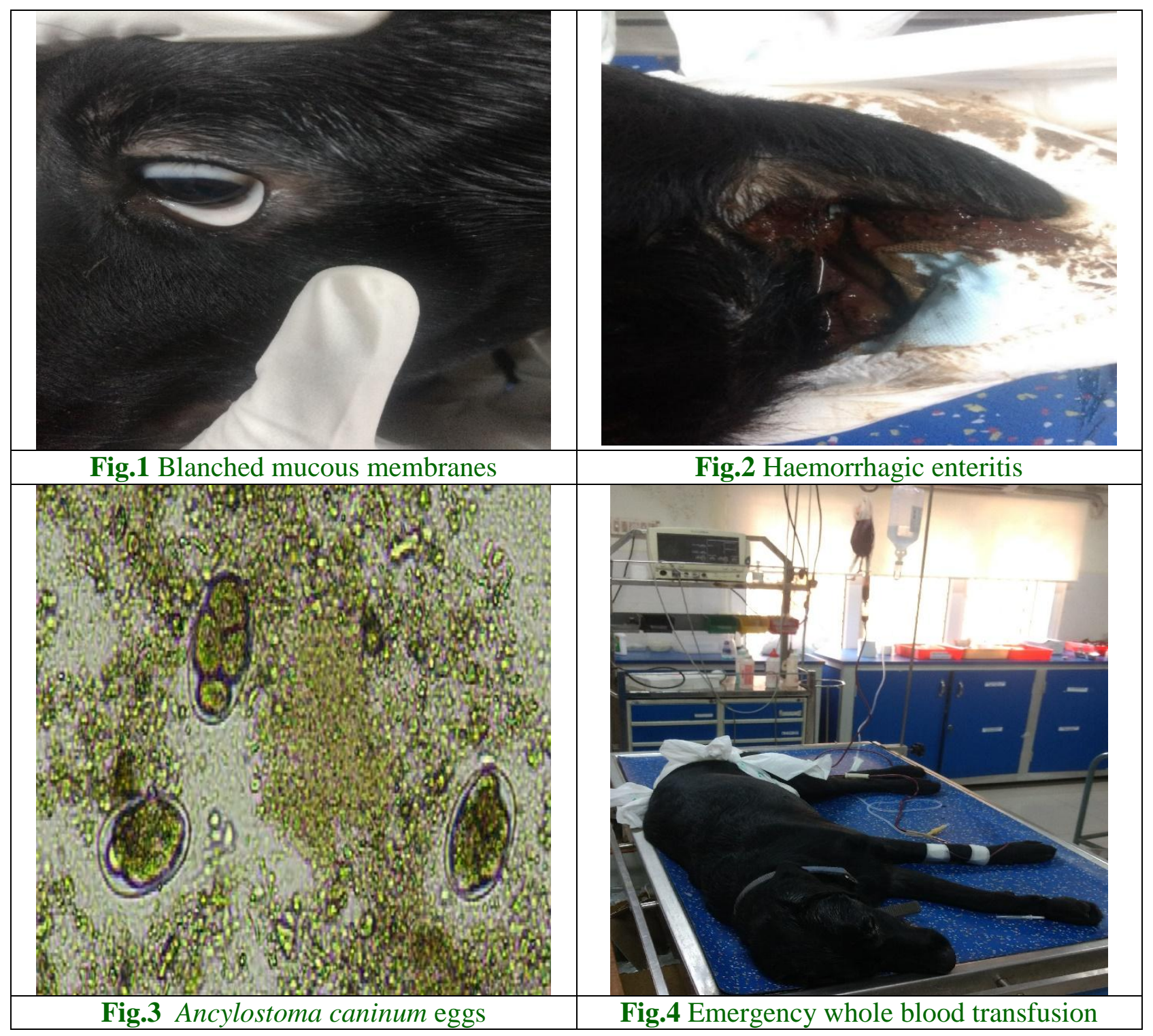

According to Helm and Knottenbelt (2014) blood transfusions are recommended when an animal has a PCV of less than 10 percent. In the present case animal had a PCV of $4.2 \%$. A combination of pyrantel pamoate and febantel reported to have very good activity against hookworms (Georgi, 2003). The role of good kennel management practices and disposal of litter should be emphasized to pet owners as a preventive measure. The client education regarding the deworming has been felt very important from this case study because the clients may not be aware of the risks of parasitic zoonotic diseases from pet dogs.
A clinical case of acute anaemia induced by Ancylostoma caninum in a puppy and its successful therapeutic management with whole blood transfusion emphasis the significance of blood transfusion in saving the precious lives of young dogs during acute anaemia induced by the voracious blood sucking canine hook worm, A. caninum.

\section{References}

Barakat, M., N. Ibrahim and A. Nasr, 2012. In Vivo Endoscopic Imaging of AncylostomosisInduced 
Gastrointestinal Bleeding: Clinical and Biological Profiles. Am. J. Trop. Med. Hyg. 87(4):701-705.

Bowman, D.D., S.P. Montgomery, A.M. Zajac, M.L. Eberhard and K.R. Kazacos, 2010. Hookworms of dogs and cats as agents of cutaneous larva migrans. Trends Parasitol. 26: 162- 7.

Cappello, M., G.P. Vlasuk, P. Bergum, S. Huang and P.J. Hotez, 1995. Ancylostoma caninum Anticoagulant Peptide: a hookworm-derived inhibitor of human coagulation factor Xa. Proc Natl Acad Sci USA. 92:6152-6.

Don, T.A., M.K. Jones, D. Smyth, P. Donoghue, P. Hotez and A. Loukas, 2004 A pore-forming haemolysin from the hookworm, Ancylostoma caninum. Int J Parasitol.34:1029- 1035. Georgi, J.R., 2003. Helminths, Chapter 3 in: Parasitology for Veterinarians.8th Edition.pp.177.

Helm, J and C. Knottenbelt, 2010. Blood transfusions in dogs and cats 1 . Indications. In Practice. 32: 184-189.

Jubb, Kennedy and Palmer. (2016) Alimentary System, Chapter 1 in: Pathology of Domestic AnimalsVolume 2.pp.214.

Yavuzer, K., A.K. Muharrem and A.S. Karadag, 2010. A Case Report of cutaneous Larva Migrans. Eurasian $J$ Med. 42:40-1.

\section{How to cite this article:}

Jasmin. R., M, Balagangatharathilagar, R. Edith and Prathaban. S. 2020. Acute Anaemia Induced by Ancylostomosis in a Puppy and its Successful Therapeutic Management - A Case Report. Int.J.Curr.Microbiol.App.Sci. 9(05): 1337-1340.

doi: https://doi.org/10.20546/ijcmas.2020.905.149 